\title{
Twenty Years of Speckle Interferometry
}

\author{
WILLIAM I. HARTKOPF \\ Center for High Angular Resolution Astronomy, Georgia State University, \\ Atlanta, GA 30303, USA
}

\section{INTRODUCTION}

The purpose of my talk is to briefly review the results of two decades of astrometric research using the technique of speckle interferometry. Although speckle, invented by Labeyrie in 1970 (Gezari et al. 1972), is the most well-known and widely-used interferometric technique in the visible and near infra-red, it neither the only technique in use, nor was it the first. Karl Schwarzschild made the first interferometric measurements of binary stars in 1895 , using the then-new technique of Michelson interferometry and following a suggestion by Michelson himself that his technique was amenable to binary star astrometry. From 1919 to 1921, Anderson (1920) and Merrill (1922) made a series of measurements of Capella and other bright stars, using a Michelson interferometer of 20-foot baseline, mounted on the Mount Wilson 100-inch. These early measurements have definitely stood the test of time. Figure 1 shows a recently calculated orbit of Capella (Bagnuolo \& Hartkopf 1989), using data spanning some 250 full revolutions. Anderson and Merrill's measurements agree beautifully with observations made 7 decades later. As a result, Capella has probably the most accurately known orbit of any visual binary. Orbital elements calculated in 1981 (McAlister 1981 ) and again 10 years later differ in period by about 14 seconds, or less than 2 parts per million!

Visual interferometric techniques were used by a handful of observers, including most notably the outstanding work of Finsen, as well as that of Jeffers, Wilson, and others, from the 1930's through the 1960's. A major improvement in the Michelson technique was realized in the photoelectric version devised by Wickes \& Dicke (1973). Tokovinin (1979) developed a modified version of the Wickes-Dicke interferometer, which he refers to as a "phase-grating stellar interferometer", in 1978, and which has been a most productive instrument for nearly 15 years now. The late 1960's also saw the expansion of Michelson and other interferometric techniques to more widely spaced multi-aperture systems, such as the Narrabri, Pulkova, and later CERGA interferometers. See McAlister (this Colloquium) for a discussion of these instruments.

Table 1 lists all single-telescope interferometry instruments known to me, with approximate dates of operation, telescopes used, and principal observers. See McAlister (1985) and McAlister \& Hartkopf (1988) for references to these observational efforts. 


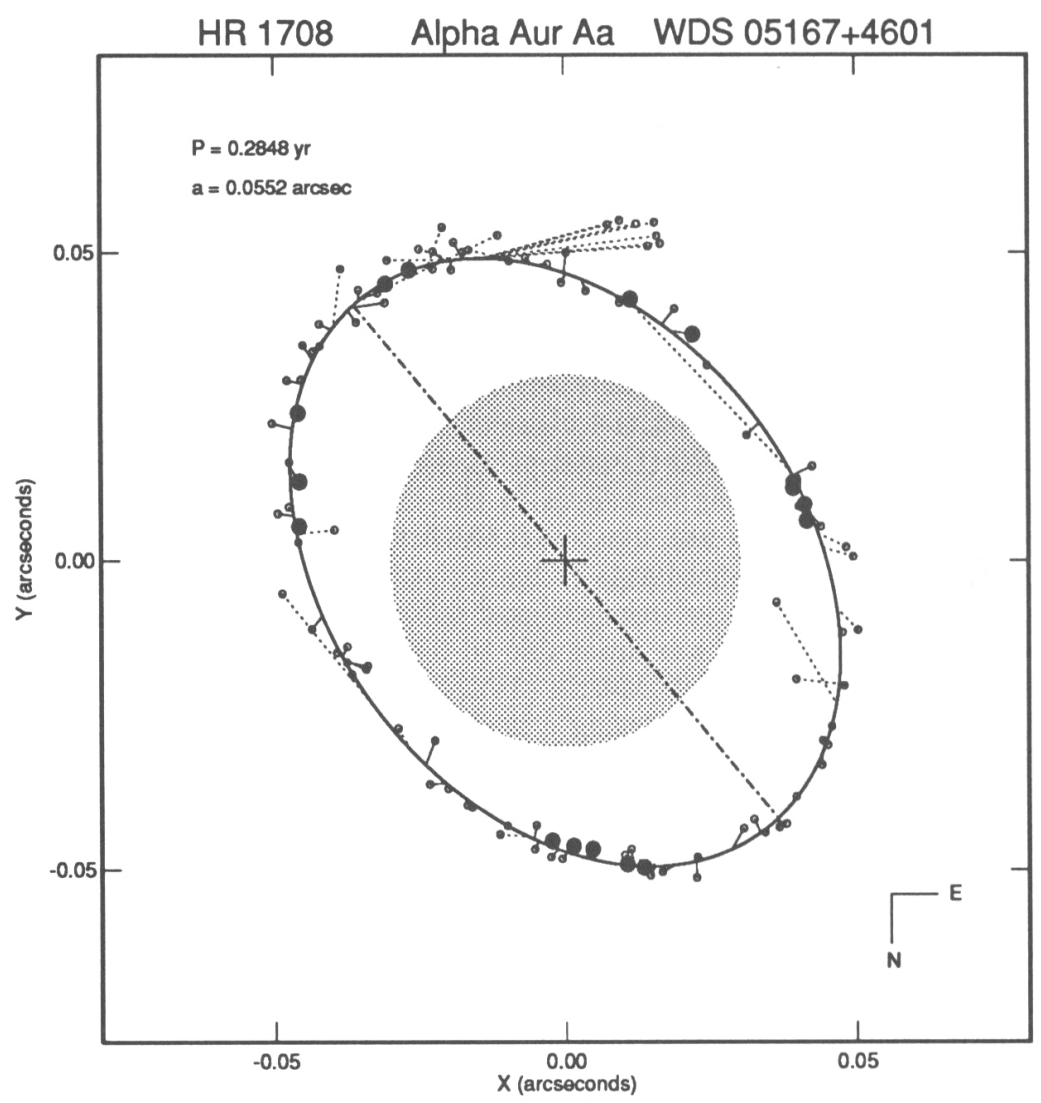

FIGURE 1. Speckle orbit of the interferometric binary Capella. The larger filled circles indicate measures from Anderson (1920) and Merrill (1922); more recent speckle measures (see McAlister \& Hartkopf 1988) are shown as smaller points.

\section{OBSERVABLE SEPARATION RANGE}

Speckle interferometry has matured over the past $20+$ years into a reliable standard technique for making relative astrometric measurements of close visual binaries - and by "close" I mean the following: The lower limit to the observed binary star separation is set by the diffraction limit of the telescope - about 30 mas for a $4-\mathrm{m}$ telescope, or about $0 " 1$ for a 1 -meter class instrument. The upper limit is set by the atmosphere, more specifically by the size of the turbulent cells in the column of atmosphere through which one is viewing. This "isoplanatic patch" typically gives a maximum observable binary separation of a few arcseconds. In practice we rarely observe stars separated by more than an arcsecond, and typically work in the range $0^{\prime \prime} .1$ to 0 ".3. Figure 2 shows the number of observations as a function of separation for all measures in our current interferometric catalog. 
TABLE 1. Interferometrists Past \& Present

\begin{tabular}{|c|c|c|c|}
\hline Schwarsschild & 1895 & Michelson interf. & Munich 10 -in \\
\hline Anderson, Merrill & $1919-1921$ & Michelson interf. & $\begin{array}{l}\text { Mt. Wilson 2.5-m } \\
\text { (6-m baseline) }\end{array}$ \\
\hline Maggini & $1922-1923$ & eyepiece interferometer & Catania 13-in \\
\hline Finsen & 1933 & Michelson interf. & Johannesburg 26-in \\
\hline Wilson & $\begin{array}{l}1934-1935 \\
1949-1954\end{array}$ & eyepiece interferometer & Flower 18-in \\
\hline Jeffers & $1939-1941$ & eyepiece interferometer & Lick 36-in \\
\hline Finsen & $1954-1969$ & eyepiece interferometer & Johannesburg 26-in \\
\hline Gesari, Labeyrie & $1970-1973$ & speckle (photographic) & $\begin{array}{l}\text { Hale 5-m, KPNO 4.0-m, } \\
\text { KPNO 2.1-m }\end{array}$ \\
\hline Wickes, Dicke & $1972-1974$ & Michelson interf. & $\begin{array}{l}\text { Mt. Wilson 1.5-m } \\
\text { Mt. Wilson 0.6-m }\end{array}$ \\
\hline $\begin{array}{l}\text { Morgan, Scaddan, } \\
\text { Tango }\end{array}$ & $1975-1978$ & speckle (photographic) & $\begin{array}{l}\text { Isaac Newton 2.5-m, } \\
\text { SAAO 1.9-m, RGO 1-m }\end{array}$ \\
\hline McAlister & $1975-1981$ & speckle (photographic) & KPNO 4-m \& 2.1-m \\
\hline $\begin{array}{l}\text { Blasit, Bonneau, } \\
\text { Koechlin }\end{array}$ & $1975-1986$ & $\begin{array}{l}\text { speckle } \\
\text { (photon-counting TV) } \\
\text { (also ICCD in 1986) }\end{array}$ & $\begin{array}{l}\text { SAO 6-m, Hale 5-m, } \\
\text { CFH 3.6-m, ESO 3.6-m, } \\
\text { Pic du Midi 2-m, } \\
\text { Haute-Provence } 1.9-\mathrm{m}\end{array}$ \\
\hline Schmidt, Angel & 1976 & 1-D speckle (SIT TV) & Steward 2.3-m \\
\hline Tokovinin & 1977 & 2-point speckle & Crimea $1.25-\mathrm{m}$ \\
\hline Balega, Dudinov & $1977-1980$ & speckle (photographic) & SAO 6-m \\
\hline $\begin{array}{l}\text { Weigelt, } \\
\text { Ebersberger }\end{array}$ & $1977-1983$ & speckle (photographic) & $\begin{array}{l}\text { ESO 3.6-m \& } 1.5-\mathrm{m} \text {, } \\
\text { Asiago 1.8-m, } \\
\text { Hoher List 1-m }\end{array}$ \\
\hline Tokovinin & $1978-$ & $\begin{array}{l}\text { photoelectric } \\
\text { phase-grating } \\
\text { interferometer }\end{array}$ & $\begin{array}{l}\text { Crimea 2.6-m, } \\
\text { Mt. Sanglok 1-m, } \\
\text { Shternberg } 0.7-\& \text { 0.6-m }\end{array}$ \\
\hline Cocke, Hege & $1979-1981$ & speckle (photographic) & $\begin{array}{l}\text { KPNO 4-m, } \\
\text { Steward 2.3-m }\end{array}$ \\
\hline $\begin{array}{l}\text { Morgan, Vine, } \\
\text { Argue }\end{array}$ & $1981-1988$ & speckle (Plumbicon) & $\begin{array}{l}\text { AAT 3.9-m, } \\
\text { Mt. Stromlo 1.9-m, } \\
\text { Lowell 1.8-m }\end{array}$ \\
\hline $\begin{array}{l}\text { McAlister, } \\
\text { Hartkopf }\end{array}$ & $1981-$ & speckle (ICCD) & $\begin{array}{l}\text { KPNO 4-m, CTIO 4-m, } \\
\text { CFH 3.6-m, Lick 3-m, } \\
\text { Mt. Wilson 2.5-m, } \\
\text { Lowell 1.8-m }\end{array}$ \\
\hline Balega & 1983- & spectle (intensified TV) & SAO 6-m \\
\hline $\begin{array}{l}\text { McCarthy, Leinert, } \\
\text { Mariotti }\end{array}$ & $1985-$ & 1-D IR speckle & $\begin{array}{l}\text { KPNO 4-m, ESO 3.6-m, } \\
\text { Calar Alto 3.5-m, } \\
\text { Steward 2.3-m }\end{array}$ \\
\hline Yiming, Yaohui & 1986 & speckle & Yunnan 1-m \\
\hline Zinnecker, Perrier & $1986-$ & 1-D IR speckle & ESO 3.6-m \\
\hline Isobe & 1987 & $\begin{array}{l}\text { speckle (intensified } \\
\text { MOS camera) }\end{array}$ & $\begin{array}{l}\text { San Pedro Martir 2.1-m } \\
\text { Shternberg 0.7- \& } 0.6-\mathrm{m}\end{array}$ \\
\hline Bakhtin & 1987 & speckle? & Abastumani AZT-11 \\
\hline $\begin{array}{l}\text { Beckers, McCarthy, } \\
\text { Henry }\end{array}$ & $1987-$ & 2-D IR speckle & $\begin{array}{l}\text { KPNO 4-m, } \\
\text { Steward 2.3-m }\end{array}$ \\
\hline Morgan, Horch & $1988-$ & speckle (MAMA) & $\begin{array}{l}\text { ESO 3.6-m, Lick 3-m, } \\
\text { Steward 2.3-m }\end{array}$ \\
\hline Worley, Douglass & $1990-$ & speckle (ICCD) & USNO 0.7-m \\
\hline Ghes & $1990-$ & 2-D IR speckle & Hale 5-m \\
\hline
\end{tabular}




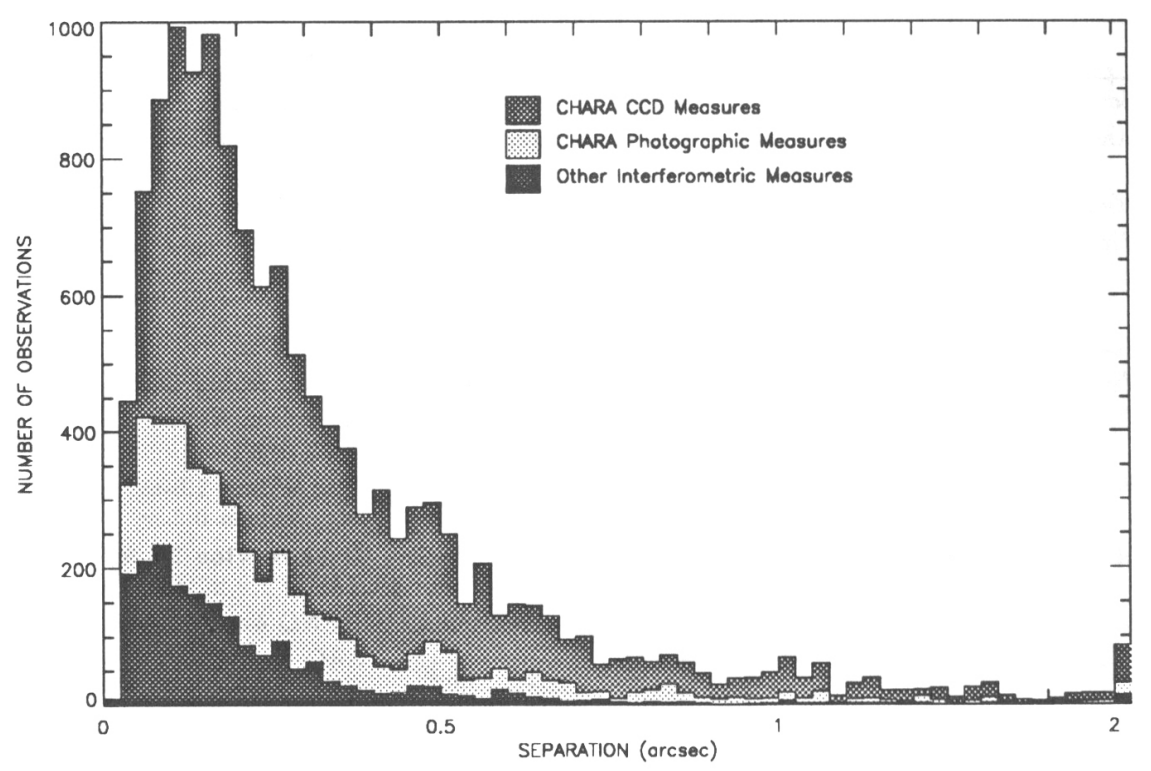

FIGURE 2. Number of speckle measures through mid-1991, as a function of separation.

\section{ACCURACY}

Speckle has shown itself to be capable of considerable accuracy, but as with any measurement technique it demands careful calibration. We have found that the use of a double slit mask, placed in the converging beam of the telescope, provides an excellent calibration of both position angle and separation. We are in effect performing a Young double-slit experiment here; the autocorrelation of speckle frames created when a single star is observed through the mask produces a set of peaks whose orientation and spacing depend solely on the geometry of the mask, effective wavelength of the filter bandpass, and focal length of the telescope. Other calibration methods may also be used, such as, for example, star trails to define an east-west axis for $\theta$.

We have found that for brighter systems with optimum separations (a few tenths of an arcsecond) and small magnitude differences, we are able to routinely reach accuracies of as little as 1 milliarcsecond. To illustrate this, Figure 3a shows an orbit for the close visual system Burnham 1099; speckle data are shown as filled circles. A residual "wave" in the speckle data, magnified in Figure 3b, was found to be due to an unseen astrometric companion, discovered independently by Frank Fekel in residuals to a 4.2-day spectroscopic component of the system. A joint analysis (Cole et al. 1992) has led to a mass estimate for this $\mathrm{Bc}$ component of 2.4 to $2.8 \mathcal{M}_{\odot}$. An interesting side note - the 4.2-day, 4.8-year, and 83-year orbits may all be coplanar. 

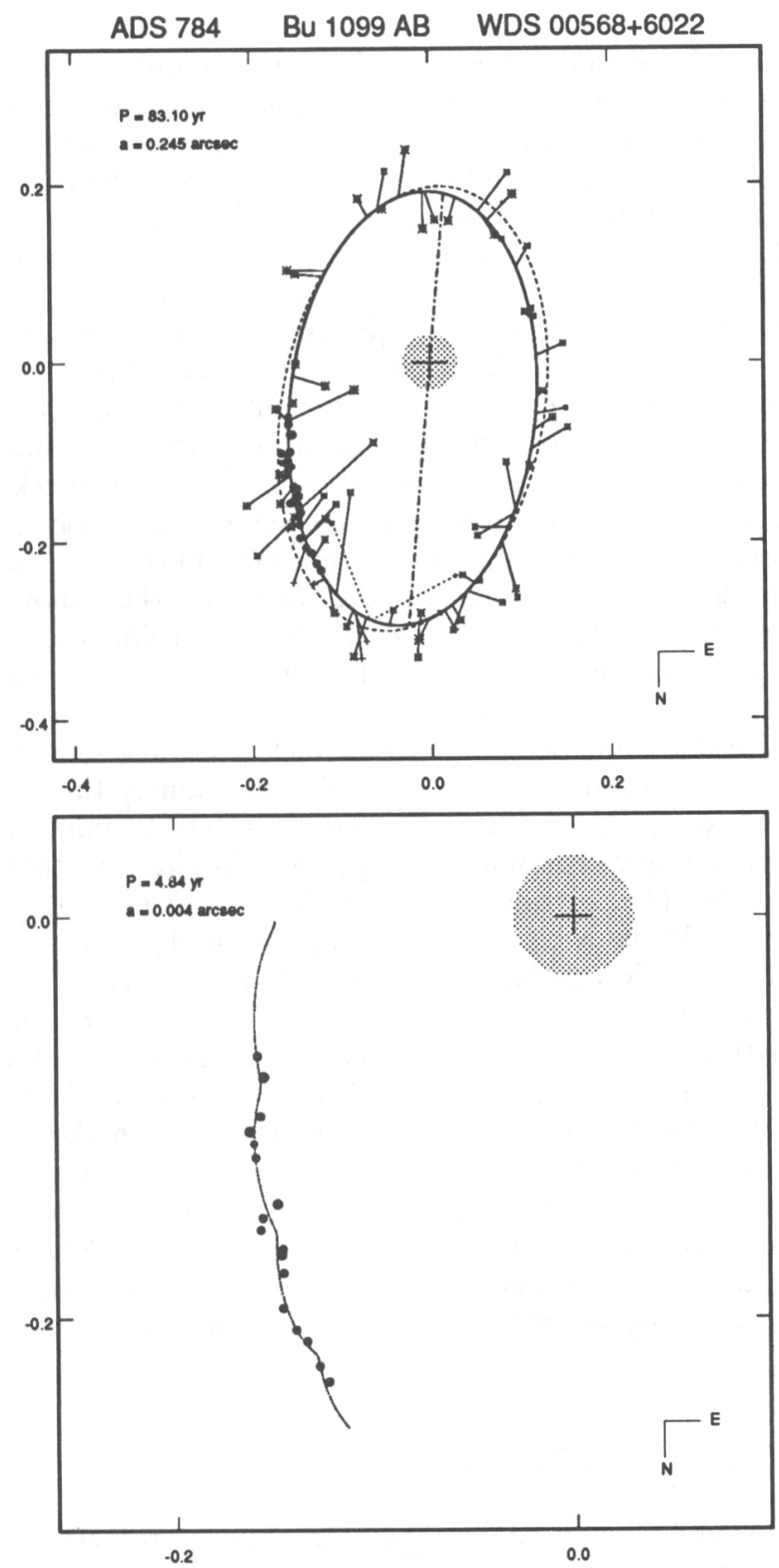

FIGURE 3. (top) Visual orbit of the long-period system Bu $1099 \mathrm{AB}$. Visual observations are indicated by plus signs or asterisks (for "small" or "large" telescope apertures, respectively. CHARA speckle observations are shown as filled circles. Axes are in seconds of arc. (bottom) A portion of the long-period orbit, perturbed by the 5-year astrometric orbit. Speckle "normal" points are indicated by filled circles. 


\section{REDUCTION TECHNIQUES}

Just as detectors have changed greatly from the photographic cameras of 20 years ago to the intensified CCD's of today, so too have the reduction techniques evolved. I arrived at Georgia State at the very end of the "photographic era" (a.k.a. the "Silver Age") and took part in some of the last reductions via the use of optical transforms. This reduction technique involved as many as 5 separate photographic steps, so although the astrometric accuracy of the data was preserved, any possible photometric content was lost.

The most common reduction technique used with 2-dimensional digital data is the "vector-autocorrelation" function, in which relative positions of all bright pixels in a frame of speckle data are mapped onto a new array. An advantage of the vector autocorrelation is its very rapid and straightforward method of calculation. A modern PC is able to process $128^{2}$ frames of speckle data in real time at standard video rates of 30 frames per second. The major disadvantage is the $180^{\circ}$ ambiguity in position angle which results from the fact that all intensity information in the pixel is ignored - the pixel is either above an intensity threshold or below it - either a " 1 " or a " 0 ". If one considers pixels at locations " $A$ " and " $B$ ", for example, both the relative position " $A-B$ " and " $B-A$ " will be added to the autocorrelation array.

Our own speckle reduction now uses a technique just one step more complicated than the standard vector autocorrelation, namely the "directed vector autocorrelation", or DVA. In the DVA routine, intensity information is saved long enough to indicate which of a pair of pixels is brighter, so that only the correct relative position (i.e. either "A-B" or " $\mathrm{B}-\mathrm{A}$ " but not both) will be added to the DVA array. While a bit (or more precisely, a "byte") more complicated than the VA, it does allow us to remove the $180^{\circ}$ ambiguity in most cases.

Other algorithms, such as "Shift-and-Add", "Fork", et cetera have also been used in attempts to go beyond simply defining a correct quadrant and actually derive differential photometric indices from speckle data. It was originally thought that the advent of digital CCD data would make this a relatively simple task, but such has not proven to be the case. Careful flat fielding and debiasing of each speckle frame are required before further processing (and in fact are done now in our DVA routine), but the whole problem of photometric calibration remains a difficult one. See Bagnuolo et al. and Barry et al. (both in this Colloquium) for descriptions of the DVA routine and speckle photometry, respectively.

\section{RESULTS TO DATE}

Since 1984 CHARA has maintained a catalog of interferometric measurements of binary stars containing all measurements published worldwide (McAlister \& Hartkopf 1984, 1988). Results as of mid-1991 are summarized in Table 2. The median separation has increased somewhat since our first catalogue, largely due to the addition to our observing lists of several thousand close visual binary stars from the Washington Double Star Catalogue (WDS), maintained at the U.S. Naval Observatory by Charles Worley. 
TABLE 2. Speckle Results as of mid-1991

\begin{tabular}{|lc|}
\hline Number of Measures & 13,452 \\
Number of Systems Resolved & 2,980 \\
Approximate Separation Range & $0^{\prime \prime} .02-7^{\prime \prime}+$ \\
(plus a few special cases outside this range) \\
Number of Negative Results & 3,451 \\
(1716 stars never resolved) & \\
Median Separation & $0 " .23$ \\
Number of Interferometric Systems & $\sim 300$ \\
\hline
\end{tabular}

\section{THE FUTURE OF SPECKLE}

Several years ago at a similar IAU meeting (Hartkopf 1991), I gave a brief review of speckle and bemoaned two major deficiencies in the speckle work being done. The first such deficiency was the lack of adequate declination coverage (although I realize that this is not unique to speckle, but has historically been a problem with standard visual and spectroscopic work as well). Virtually no work was being done in the southern hemisphere. Binary stars discovered in some cases 50 years ago had never even been confirmed, while some regions of the sky had never even been surveyed systematically for new binaries. Figure 4 illustrates the problem - as of late 1988 there were some 44 measures for stars south of $-30^{\circ}$, versus over 3,600 measures for stars north of $+30^{\circ}$. Routine speckle observations were begun by us in the southern hemisphere in 1989, however, and the situation is slowly being remedied. Also shown in this figure are the number of observations made through mid-1991. Although the southern skies are still

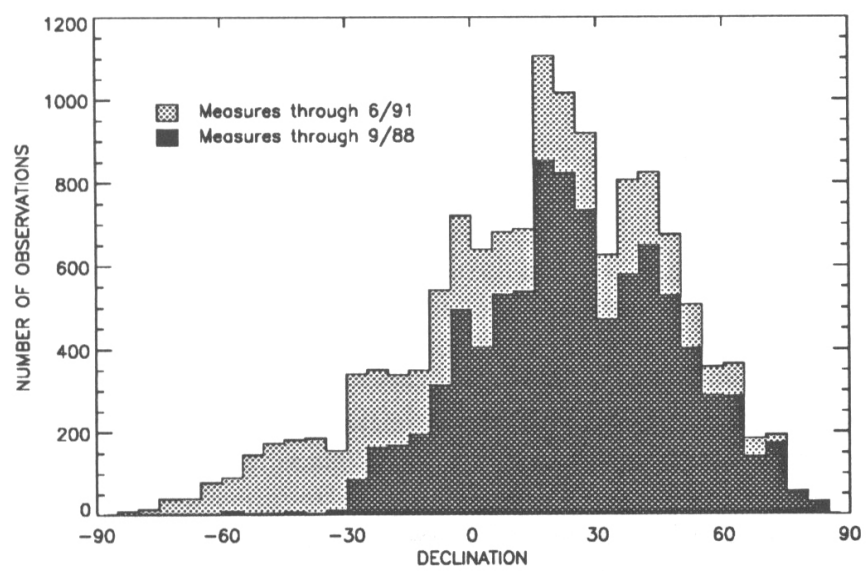

FIGURE 4. Number of speckle measures, as a function of declination. The darker shaded region indicates the distribution through September 1988, the lighter region the improvement in this distribution after regular southern hemisphere observations were begun in early 1989 . 


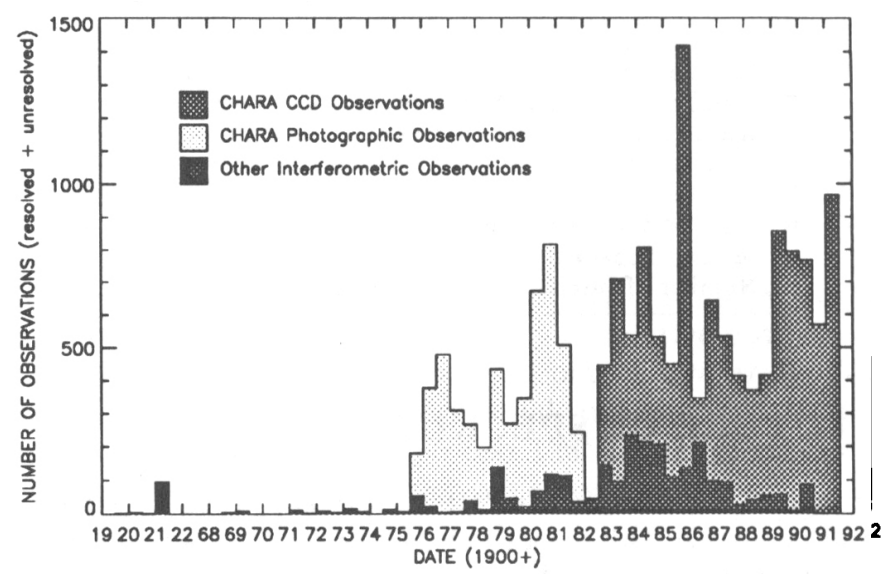

FIGURE 5. Number of speckle measure as a function of time. Note the pronounced gap in early 1982 .

woefully underrepresented, there has been considerable improvement. The number of binaries south of $-30^{\circ}$ declination has increased during this 2.5-year period by a factor of 25 .

The second deficiency I noted was simply a shortage of speckle observers. Figure 5 illustrates my point. Throughout the late 1970's and 1980's total output of speckle data amounted to about 1,000 observations per year, with one notable exception. In early 1982 the CHARA speckle group retired an old photographic speckle camera in favor of its current ICCD system. As a result of the time spent in engineering tests, et cetera, we took no speckle data during the first half of the year. The worldwide output of speckle measurements during that entire 6month period was 26 . The point - there was simply too much dependence on a single speckle setup. Although this figure indicates little change in the situation thus far, I believe that considerable improvement is finally underway. The U.S. Naval Observatory now has a well-functioning speckle camera, as described by Worley \& Douglass (this Colloquium). A group of astronomers in Korea has contracted for similar equipment, now under construction, and other groups in Spain and the U.S. are looking into the possibility of getting into the field, as well. Turner et al. (this Colloquium) even describe an inexpensive speckle camera which may be within the price range affordable to a serious amateur astronomer.

The long-term future of speckle is more uncertain. The rapid advances being made now in adaptive optics, laser guide stars, and the like probably means that before the end of the decade most large telescopes will be able to routinely remove most atmospheric distortion before the science data are collected, making speckle unnecessary. Barring a real breakthrough which brings the cost of such equipment down sharply, I don't believe that more moderate-sized telescopes will be outfitted with adaptive optics for several years beyond that. I think for the present we'll have to worry more about our speckle cameras wearing out than about them becoming obsolete. 


\section{ACKNOWLEDGEMENTS}

Speckle interferometric studies of binary stars at Georgia State University have been made possible since the program began in 1977 by the continuous support of the National Science Foundation and by allocations of telescope time at the 4-m telescope on Kitt Peak and, since 1989, the 4-m telescope on Cerro Tololo. We are to grateful NSF and NOAO for their generosity.

\section{REFERENCES}

Anderson, J.A. 1920, $A p J, 51,263$

Bagnuolo, W.G. Jr. \& Hartkopf, W.I. 1989, $A J, \mathbf{9 8 ,} 2275$

Cole, W.A., F.C. Fekel, F.C., Hartkopf, W.I., McAlister, H.A., \& Tomkin, J. 1992, AJ, 103, 1357

Gezari, D.Y., Labeyrie, A., \& Stachnik, R.V. 1972, ApJ, 173, L1

Hartkopf, W.I. \& McAlister, H.A. 1991, Fundamentals of Astrometry, International Astronomical Union Colloquium 100, ApESS, 177, 161

McAlister, H.A. 1981, $A J, 86,795$

McAlister, H.A. \& W.I. Hartkopf, W.I. 1984, Center for High Angular Resolution Astronomy, Contribution No. 1.

McAlister, H.A. \& W.I. Hartkopf, W.I. 1988, Center for High Angular Resolution Astronomy, Contribution No. 2.

Merrill, P.W. 1922, ApJ, 56, 43

Tokovinin, A.A. 1979, Sov. Astr. Lett., 5, 229

Wickes, W.C. \& Dicke, R.H. 1973, $A J, 78,757$

\section{DISCUSSION}

HEINTZ: The scatter in the graph of visual observations is much enhanced by the plotting of $\rho(\theta)$. The more relevant function $\theta(\mathrm{t})$ will show much smaller scatter.

KAROVSKA: I have three questions. First, what is the precision in the $\Delta \mathrm{m}$ measurements? Second, how do you measure $\Delta \mathrm{m}$ and its errors? Third, how do you measure the position of the binary and is it (and by how much) affected by the distortion introduced by the instrument residual dispersion and the focus changes of the telescope.

HARTKOPF: As I said, photometric calibration of our system remains a major problem, so our $\Delta \mathrm{m}$ estimates are very rough - certainly no better than 0.1 mag. The technique used most often is Bagnuolo's "fork" algorithm. For measuring the system geometry, the background is first subtracted from the vector autocorrelation or DVA using a simple boxcar or Gaussian smoothing technique. Using an interactive program, we pick an approximate peak position, then the program picks a small array of pixels centered on that approximate peak and makes a paraboloidal fit to determine a centroid. We have not noticed any substantial positional distortions in our array, although Douglass \& Worley note in their poster that we may have some small systematic error for separations greater than one arcsecond. We have a limited number of observations of systems that wide, however, so we haven't done a detailed study. 
MCALISTER: Concerning the accuracy of our differential photometry, we presently consider our results qualitative rather than quantitative. While we may have a precision of $\pm 0.1 \mathrm{mag}$, we worry about significant systematic errors that greatly degrade this. We are actively working on this important problem. The limiting accuracy of our astrometry is ultimately set by the uncertainty in the telescope focal length, known to about $\pm 0.5 \%$.

WORLEY: In answer to Karovska, there are two philosophies in speckle. In the first case, there are groups that measure large numbers of binaries. The proof of what they do (without elaborate worries about secondary effects) is how well their measures represent an elliptical orbit. On the other hand, there are groups that make a few measures of almost unique objects. I would like to see them make observations of a larger list of stars which the rest of us observe, so that we can see if their observations really measure up.

ISOBE: Don't you use any reference stars located near each object? If you do, you can reduce much of the central spiky portion of the Fourier transform and get higher precision of measurement of binary separations.

HARTKOPF: No, we haven't found the use of reference stars necessary or really feasible. We work in speckle on a "wholesale" basis - as many as 300 observations in a given night. We also find that seeing variations over small repointing angles and over short time intervals make the use of reference stars far from the ideal situation as depicted by classical speckle interferometry.

ZINNECKER: Which magnitude limits and which dynamical range do you reach? Did you apply any bispectrum analysis and what is your experience with it?

HARTKOPF: I don't personally have any experience with bispectrum analysis. Our magnitude limit has actually declined during the past several years, due to the aging intensifier used with our RCA CCD. Limiting magnitude for that camera is about 9 , while we were able to see a $15^{\text {th }}$ magnitude pair with it back in 1983 (although we typically didn't go fainter than about 11 or 12). We have a new ITT CCD camera which has a better limit (perhaps 11) but haven't reduced enough data to have a good feel for what the value is. As for dynamical range, we've reached perhaps 3 magnitudes of $\Delta \mathrm{m}$, although this is, of course, a function of stellar brightness, separation, and seeing.

VAN DESSEL: What has been the contribution of lunar occultation binaries to your program, and how do their results compare to yours?

HARTKOPF: The lists of occultation binaries have provided a fair number of objects for our observing lists. We have resolved a number of occultation pairs by speckle but have not as yet done any comparative study. 